\title{
Modeling and Validation of an Open-Source Mean Value Heavy-Duty Diesel Engine Model
}

\author{
Kristoffer Ekberg ${ }^{*}$, Viktor Leek, Lars Eriksson
}

Vehicular Systems, Department of Electrical Engineering, Linköping University, Sweden, 58183 Linköping; *kristoffer.ekberg@liu.se

SNE 28(4), 2018, 197-204, DOI: 10.11128/sne.28.tn.10451

Received: November 27, 2018; Revised December 10, 2018; Accepted: December 12, 2018

SNE - Simulation Notes Europe, ARGESIM Publisher Vienna, ISSN Print 2305-9974, Online 2306-0271, www.sne-journal.org

Abstract. The pursuit of lower fuel consumption and stricter emission legislation has made a simulation- and optimization-based development methodology important to the automotive industry. The keystone in the methodology, is the system model. But for the results obtained using a model to be credible, the model has to be validated. The paper validates an open-source, meanvalue engine model of a 13 liter $\mathrm{Cl}$ inline 6 cylinder heavyduty engine, and releases it as open-source.

\section{Introduction}

In today's automotive industry, there is a drive for lower fuel consumption and better control of emissions. Simulation and numerical optimization are two tools that can be used to achieve that. The keystone in a simulation driven approach, is the system model. For it (and the results obtained using it) to be credible, it needs to be validated. This paper presents the model structure of an open-source engine model and validates it.

The engine is a 13 liter CI inline 6 cylinder Scania engine. The model structure is a mean-value engine model (MVEM) [5], this type of structure does not model the piston movement explicitly. Instead the mean flow in and out of the cylinders is modeled. This makes the model computationally efficient, and suitable for control and optimization of the air and fuel path of the engine. It has a low number of states for efficient simulations, and is continuously differentiable for use with gradient-based optimization solvers.

The model is a continuation of previous modeling work by the authors, described in [1]. In contrast to the previous work, indicated mean effective pressure
(IMEP), pump mean effective pressure (PMEP) and friction mean effective pressure (FMEP), have been remodeled, the compressor model has been changed for a more advanced model presented in [7], the turbine has been remodeled to better describe the efficiency, and the model is validated as a complete system.

The purpose of this paper is to provide an open model to the research community, and to invoke confidence in the model by validating it. The paper is outlined as follows. In Section 1 the data sets are presented, in Section 2 the estimation criterion is presented, in Section 3 the model equations are presented and the submodels are validated, in Section 4 all submodels are connected and the complete modeled is tuned and validated against measurement data, and in Section 5 the model is simulated and its basic simulation properties are presented.

Contribution. The main contribution of this paper is a validated open-source MVEM of a Scania 13 liter 6 cylinder engine, downloadable from www.fs.isy.liu.se/Software/. Also, new component models for the engine torque, and an adaptation of an existing turbine model are introduced.

\section{Data}

Six datasets have been used for modeling and validation, see Table 1. Dataset A is dynamometer data of the engine, and is the primary modeling set. Dataset $\mathrm{B}$ is simulation data from a model of the same engine obtained using a detailed model implemented in GTPower [4]. In B, the air-to-fuel ratio is varied for the operating points and is primarily used to develop the torque model. Dataset $\mathrm{C}$ is used to model the compressor, and dataset $\mathrm{D}$ is used for modeling the turbine. Dataset $\mathrm{E}$ is used to validate the complete model, and dataset $\mathrm{F}$ is used for throttle modeling. 


\begin{tabular}{l|l|l} 
Dataset & Signals & Samples \\
\hline A. Stationary measurement data & 24 & 235 \\
B. GT Power Simulation & 22 & 160 \\
C. Compressor map & 4 & 73 \\
D. Turbine map & 4 & 38 \\
E. Dynamic measurement data & 24 & 69598 \\
F. Throttle effective area & 1 & 11 \\
\hline
\end{tabular}

Table 1: Datasets used to find model parameters.

\section{Parameter Estimation}

To parameterize the sub-models, the following criterion is used:

$$
\theta^{*}=\arg \min _{\theta} \sum_{k=1}^{K} e_{k}^{2}(\theta)
$$

where $\theta^{*}$ are the criterion minimizing parameters, $e_{k}$ the model error at sample $k$, and $K$ the number of samples in the data set.

Some of the submodels are not modeled using dataset $\mathrm{A}$, which is the desired model behavior at steady-state. To compensate for this, the parameters need to be refitted to represent dataset $\mathrm{A}$. This is done using a regularization technique, which is selected in order to preserve the model structure, according to:

$$
\theta^{\star}=\arg \min _{\theta}\left(\sum_{k=1}^{K} e_{k}^{2}(\theta)+C \sum_{i=1}^{I}\left(\mu_{i} \frac{\theta_{i}^{*}-\theta_{i}}{\theta_{i}^{*}}\right)^{2}\right)
$$

where $\theta^{\star}$ are the parameters refitted to dataset A, $\theta^{*}$ the parameters obtained using (1) and a dataset different than A, $I$ the numbers of parameters to refit, $\mu_{i}$ the weight assigned to parameter $i$, and $C$ the cost of changing the parameters.

To evaluate the model fit, the following measures are used:

$$
\begin{aligned}
& \bar{e}=\frac{1}{K} \sum_{k=1}^{K}\left|e_{k}\right| \\
& e_{\sigma}=\sqrt{V\left[\left|e_{k}\right|\right]} \\
& \bar{e}_{\text {rel }}=\frac{1}{K} \sum_{k=1}^{K}\left|\frac{e_{k}}{s_{k}}\right| \\
& e_{\text {rel }, \sigma}=\sqrt{V\left[\left|e_{k} / s_{k}\right|\right]}
\end{aligned}
$$

where $\bar{e}$ is the mean absolute error, $e_{\sigma}$ the standard deviation of the absolute error, $\bar{e}_{\text {rel }}$ the relative error, $s_{k}$ the k:th sample, and $e_{\mathrm{rel}, \sigma}$ the standard deviation of the relative error. The variance was calculated using the command var in Matlab R2017b.

\section{Model}

In the presentation of the validated model structure, the time dependence, $(t)$, is used to distinguish variables from constants, dependence on other variables is omitted for notational simplicity.

The model has four states $x(t)$ :

$$
x(t)=\left[p_{\mathrm{c}}(t), p_{\mathrm{im}}(t), p_{\mathrm{em}}(t), \omega_{\mathrm{tc}}(t)\right]
$$

where $p_{\mathrm{c}}$ is the pressure after the compressor, $p_{\text {im }}$ the intake manifold pressure, $p_{\mathrm{em}}$ the exhaust manifold pressure, and $\omega_{\mathrm{tc}}$ the turbocharger angular velocity. The model has three control inputs:

$$
u(t)=\left[u_{\mathrm{f}}(t), u_{\mathrm{thr}}(t), u_{\mathrm{wg}}(t)\right]
$$

where $u_{\mathrm{f}}$ is the fuel mass injected per cycle, $u_{\mathrm{thr}}$ the throttle position, and $u_{\mathrm{wg}}$ the wastegate position. The engine speed, $N_{\text {ice }}$, is treated as an external input into the system. Figure 1 shows an overview of the model, where the states and control inputs are visualized.

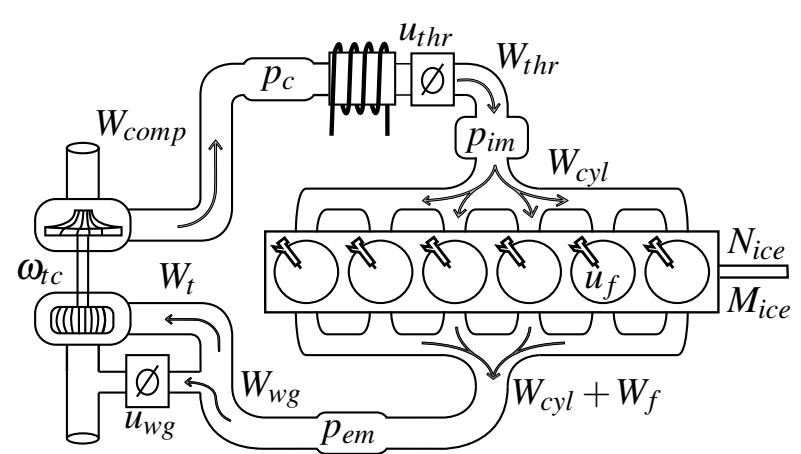

Figure 1: Model overview. Shown are the four states: pressure after the compressor $p_{\mathrm{c}}$, intake manifold pressure $p_{\text {im }}$, exhaust manifold pressure $p_{\text {em }}$ and turbocharger angular velocity $\omega_{\mathrm{tc}}$, and the three control inputs: Fuel injection per cycle $u_{\mathrm{f}}$, throttle effective area $u_{\text {thr }}$, and wastegate effective area $u_{\mathrm{wg}}$. 


\subsection{Control volumes}

The volume after the compressor, intake manifold and exhaust manifold are seen as thermodynamic control volumes, and modeled as dynamic states with filling and emptying dynamics. Using an isothermal model and by assuming mass conservation, constant $c_{\mathrm{p}}$ and $c_{\mathrm{v}}$, the dynamics are described as:

$$
\begin{aligned}
\frac{d}{d t} p_{\mathrm{c}}(t) & =\frac{R_{\mathrm{a}} T_{\mathrm{c}}(t)}{V_{\mathrm{c}}}\left(W_{c}(t)-W_{\mathrm{thr}}(t)\right) \\
\frac{d}{d t} p_{\mathrm{im}}(t) & =\frac{R_{\mathrm{a}} T_{\mathrm{im}}}{V_{\mathrm{im}}}\left(W_{\mathrm{thr}}(t)-W_{\mathrm{cyl}}(t)\right) \\
\frac{d}{d t} p_{\mathrm{em}}(t) & =\frac{R_{\mathrm{e}} T_{\mathrm{em}}(t)}{V_{\mathrm{em}}}\left(W_{\mathrm{cyl}}(t)+W_{\mathrm{f}}(t)-W_{\mathrm{t}}(t)-W_{\mathrm{wg}}(t)\right)
\end{aligned}
$$

Parameters. There are four parameters to estimate: volume after the compressor $V_{\mathrm{c}}$, intake manifold volume $V_{\mathrm{im}}$, exhaust manifold volume $V_{\mathrm{em}}$, and intake manifold temperature $T_{\mathrm{im}}$.

Parametrization and Validation. In dataset A, the mean value of the measured temperature in the intake manifold is $304.00 \mathrm{~K}$ with a standard deviation of $1.11 \mathrm{~K}$, indicating that a constant temperature in the intake manifold is an acceptable modeling assumption. The charge air cooler is assumed to be ideal, therefore the temperature in the intake manifold equals the surrounding temperature, $T_{\mathrm{im}}=T_{\mathrm{amb}}$. The sizes of the volumes $V_{\mathrm{c}}, V_{\mathrm{im}}$ and $V_{\mathrm{em}}$ are tuned until the dynamics of the model comply with the measurements. Validation of the volume sizes is seen in Section 5.

\subsection{Throttle}

In accordance with the throttle modeling approach in []MCED, the mass flow through the throttle is modeled as an isentropic compressible restriction, according to:

$$
W_{\mathrm{thr}}(t)=\frac{p_{\mathrm{c}}(t)}{\sqrt{R_{\mathrm{a}} T_{\mathrm{c}}(t)}} C_{\mathrm{D}, \mathrm{thr}} A_{\mathrm{thr}, \max } u_{\mathrm{thr}}(t) \Psi_{\mathrm{thr}}(t)
$$

where the throttle air mass flow is denoted by $W_{\text {thr }}$, the maximum throttle area by $A_{\mathrm{thr} \text { max }}$, the flow coefficient by $C_{\mathrm{D}, \text { thr }}$, the temperature by $T_{\mathrm{c}}$, and the specific gas constant of air by $R_{a}$. The throttle effective area is controlled directly via the control signal $u_{\text {thr }}(t)$. The flow parameter $\Psi_{\text {thr }}$ is taken from [9], and represented as in
[6]:

$$
\begin{gathered}
\Psi_{\mathrm{thr}}(t)=\sqrt{\frac{\gamma_{\mathrm{a}}+1}{2 \gamma_{\mathrm{a}}}\left(1-\Pi_{\mathrm{thr}}(t)\right)\left(\Pi_{\mathrm{thr}}(t)+\frac{\gamma_{\mathrm{a}}-1}{\gamma_{\mathrm{a}}+1}\right)} \\
\Pi_{\mathrm{thr}}(t)= \begin{cases}\frac{p_{\mathrm{im}}(t)}{p_{\mathrm{c}}(t)} & \text { if } \frac{p_{\mathrm{im}}(t)}{p_{\mathrm{c}}(t)} \geq \frac{1}{\gamma_{\mathrm{a}}+1} \\
\frac{1}{\gamma_{\mathrm{a}}+1} & \text { otherwise }\end{cases}
\end{gathered}
$$

where $\gamma_{a}$ is the ratio of specific heats. The conditional equation (11b) does not have a continuous derivative at the switching point. The Logistic function is therefore used to emulate the switching (11b):

$$
\begin{aligned}
\Pi_{\mathrm{thr}}(t) & =\Pi^{\text {choke }}+c_{\text {switch }}(t)\left(\Pi(t)-\Pi^{\text {choke }}\right) \\
c_{\text {switch }}(t) & =\frac{1}{1+e^{-c \Psi\left(\Pi(t)-\Pi^{\text {choke }}\right)}} \\
\Pi^{\text {choke }} & =\frac{1}{\gamma_{\mathrm{a}}+1} \\
\Pi(t) & =\frac{p_{\text {im }}(t)}{p_{\mathrm{c}}(t)}
\end{aligned}
$$

where $c \Psi$ is the steepness parameter of the Logistic function. The implemented flow parameter $\Psi_{\text {thr }}(t)$ is modelled as (11a), where $\Pi_{\mathrm{thr}}(t)$ is modelled according to (12).

Parameters. There is one parameter to estimate, $C_{\mathrm{D}, \mathrm{thr}}$. The throttle area $A_{\mathrm{thr} \text { max }}$ is known from dataset $\mathrm{F}$.

Parametrization and Validation. A dataset for validating the throttle model was not obtained. $C_{\mathrm{D} \text {,thr }}$ is seen as a tuning parameter in optimizing the steadystate levels of the model. A validation of $\Psi_{\text {thr }}$ is depicted in Figure 2.

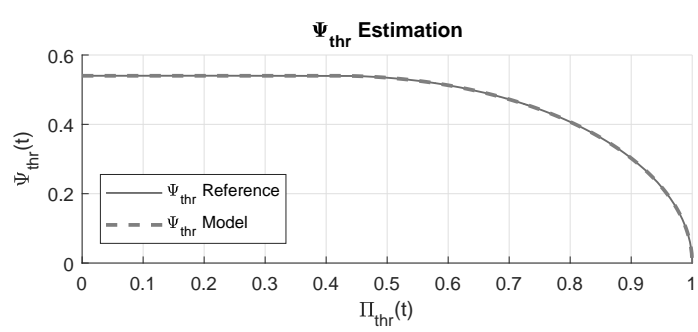

Figure 2: $\Psi_{\text {thr }}(t)$-functions where the blue solid reference is described by (11), the red trace is when $\Pi_{\mathrm{thr}}(t)$ is described according to (12). 


\subsection{Cylinder}

The cylinder air mass flow is modeled with the help of the volumetric efficiency, $\eta_{\mathrm{vol}}$, [5]. It is expressed using a single constant as in [3]:

$$
W_{\mathrm{cyl}}(t)=\eta_{\mathrm{vol}} \frac{V_{\mathrm{D}} p_{\mathrm{im}}(t)}{n_{\mathrm{r}} R_{\mathrm{a}} T_{\mathrm{im}}} \frac{N_{\mathrm{ice}}(t)}{60}
$$

The cylinder fuel mass flow is calculated from the fuel injection per cycle and engine speed:

$$
W_{\mathrm{f}}(t)=\frac{n_{\text {cyl }} N_{\text {ice }}(t) u_{\mathrm{f}}(t)}{n_{\mathrm{r}}} \frac{10^{-6}}{60}
$$

The fuel-to-air equivalence ratio $\phi(t)$ is calculated as:

$$
\phi(t)=\frac{W_{\mathrm{f}}(t)}{W_{\mathrm{cyl}}(t)} \mathrm{AF}_{\mathrm{s}}
$$

where $\mathrm{AF}_{\mathrm{S}}$ is the air-to-fuel stoichiometric ratio.

To model the engine torque $M_{\text {ice }}(t)$, it is broken down into the components: gross indicated torque $M_{\mathrm{ig}}(t)$, pumping torque $M_{\text {pump }}(t)$, and friction torque $M_{\text {fric }}(t)$, and calculated as:

$$
M_{\text {ice }}(t)=M_{\mathrm{ig}}(t)-M_{\text {pump }}(t)-M_{\text {fric }}(t)
$$

where the three components $M_{\mathrm{ig}}(t), M_{\text {pump }}(t)$ and $M_{\text {fric }}(t)$ are expressed in the normalized quantities IMEP, PMEP and FMEP [3].

The indicated torque is modeled according to:

$$
\begin{aligned}
M_{\mathrm{ig}}(t) & =\frac{V_{\mathrm{D}}}{n_{\mathrm{r}} 2 \pi} \operatorname{IMEP}(t) \\
\operatorname{IMEP}(t) & =\eta_{\mathrm{ig}}(t) \frac{q_{\mathrm{HV}} u_{\mathrm{f}}(t) 10^{-6} n_{\mathrm{cyl}}}{V_{\mathrm{D}}} \\
\eta_{\mathrm{ig}}(t) & =\left(1-\frac{1}{r_{\mathrm{c}}(t)-1}\right) \eta_{\mathrm{cal}}(t) \\
\gamma_{\mathrm{cyl}}(t) & =c_{\gamma, 0}+c_{\gamma, 1} \phi(t)+c_{\gamma, 2} \phi^{2}(t)
\end{aligned}
$$

Where the operating point dependent losses, are modeled using the following polynomial structure:

$$
\begin{aligned}
\eta_{\mathrm{cal}}(t) & =c_{\mathrm{cal}, 2}(t)\left(\frac{u_{\mathrm{f}}(t)}{100}-c_{\mathrm{cal}, 1}(t)\right)^{2}+c_{\mathrm{cal}, 0} \\
c_{\mathrm{cal}, 1}(t) & =c_{\mathrm{cal}, 10}+c_{\mathrm{cal}, 11} \frac{N_{\mathrm{ice}}(t)}{1000}
\end{aligned}
$$

$$
c_{\mathrm{cal}, 2}(t)=c_{\mathrm{cal}, 20}+c_{\mathrm{cal}, 21} \frac{N_{\mathrm{ice}}(t)}{1000}+c_{\mathrm{cal}, 22}\left(\frac{N_{\mathrm{ice}}(t)}{1000}\right)^{2}
$$

The pumping losses are modeled as:

$$
\begin{aligned}
M_{\text {pump }}(t) & =\frac{V_{\mathrm{D}}}{n_{\mathrm{r}} 2 \pi} \operatorname{PMEP}(t) \\
\operatorname{PMEP}(t) & =c_{\mathrm{PMEP}, 0}+c_{\mathrm{PMEP}, 1}\left(p_{\mathrm{em}}(t)-p_{\text {im }}(t)\right)
\end{aligned}
$$

The losses which are not included in the pumping losses are lumped into the friction term, which is modeled as a polynomial in fuel injection and engine speed:

$$
\begin{aligned}
M_{\text {fric }}(t) & =\frac{V_{\mathrm{D}}}{n_{\mathrm{r}} 2 \pi} \operatorname{FMEP}(t) \\
\operatorname{FMEP}(t) & =c_{\mathrm{f}, 0}+c_{\mathrm{f}, 1} N_{\text {ice }}(t)+c_{\mathrm{f}, 2} u_{\mathrm{f}}(t)+c_{\mathrm{f}, 3} u_{\mathrm{f}}(t) N_{\text {ice }}(t)
\end{aligned}
$$

The temperature of the gas exiting the cylinders, $T_{e}$, is modeled based on calculations on an ideal cycle and adding the parameter $\eta_{\text {sc }}$ to include non-ideal properties:

$$
\begin{aligned}
T_{\mathrm{e}}(t) & =\eta_{\mathrm{sc}} \Pi_{\mathrm{cyl}}^{1-1 / \gamma_{\mathrm{a}}}(t) r_{\mathrm{c}}^{1-\gamma_{\mathrm{a}}}\left(\frac{q_{\mathrm{in}}(t)}{c_{\mathrm{p}, \mathrm{a}}}+T_{\mathrm{im}} r_{c}^{\gamma_{\mathrm{a}}-1}\right) \\
q_{\mathrm{in}}(t) & =\frac{W_{\mathrm{f}}(t)}{W_{\mathrm{f}}(t)+W_{\mathrm{cyl}}(t)} q_{\mathrm{HV}} \\
\Pi_{\mathrm{cyl}}(t) & =\frac{p_{\mathrm{em}}(t)}{p_{\mathrm{im}}(t)}
\end{aligned}
$$

To take the heat transfer from the exhaust manifold to the surroundings into account, the mean value exhaust gas temperature model from [2] is implemented:

$$
T_{\mathrm{em}}(t)=T_{\mathrm{amb}}+\left(T_{\mathrm{e}}(t)-T_{\mathrm{amb}}\right) e^{-\frac{c_{\mathrm{em}, \mathrm{h}}}{\left(W_{\mathrm{cyl}}(t)+W_{\mathrm{f}}(t) c_{\mathrm{p}, \mathrm{e}}\right.}}
$$

Parameters. There are 18 parameters to estimate: $\eta_{\mathrm{vol}}, c_{\gamma, 0}, c_{\gamma, 1}, c_{\gamma, 2}, c_{\mathrm{cal}, 0}, c_{\mathrm{cal}, 10}, c_{\mathrm{cal}, 11}, c_{\mathrm{cal}, 20}, c_{\mathrm{cal}, 21}$, $c_{\mathrm{cal}, 22}, c_{\mathrm{f}, 0}, c_{\mathrm{f}, 1}, c_{\mathrm{f}, 2}, c_{\mathrm{f}, 3}, c_{\mathrm{PMEP}, 0}, c_{\mathrm{PMEP}, 1}, \eta_{\mathrm{sc}}$, and $c_{\mathrm{em}, \mathrm{h}}$.

Parametrization and Validation. To preserve the properties observed in the data, the parameterization is carried out in steps. The cylinder massflow is estimated using dataset A. The error function is calculated as:

$$
e_{k, \eta \mathrm{vol}}=\left(\eta_{\mathrm{vol}, \mathrm{data}, k}-\eta_{\mathrm{vol}}\right)^{2}
$$

The resulting fit is shown in Table 2. 
The indicated torque is estimated using data set B (Table 1). The parameterization was done by minimizing the following error function:

$$
\begin{aligned}
& e_{k, \mathrm{IMEP}}=\left(\operatorname{IMEP}_{\mathrm{data}, k}-\operatorname{IMEP}_{\text {model }, k}\left(\theta_{\mathrm{IMEP}}\right)\right)^{2} \\
& \theta_{\mathrm{IMEP}}=\left[c_{\gamma, 0}, c_{\gamma, 1}, c_{\gamma, 2}, c_{\mathrm{cal}, 10}, c_{\mathrm{cal}, 11}, c_{\mathrm{cal}, 20}, c_{\mathrm{cal}, 21}, c_{\mathrm{cal}, 22}\right]
\end{aligned}
$$

The resulting fit is shown in Table 3 .

The pumping torque is estimated using data set B (Table 1). The parameterization was done by minimizing the following error function:

$$
\begin{aligned}
e_{k, \mathrm{PMEP}} & =\left(\mathrm{PMEP}_{\mathrm{data}, k}-\mathrm{PMEP}_{\text {model }, k}\left(\theta_{\mathrm{PMEP}}\right)\right)^{2} \\
\theta_{\mathrm{PMEP}} & =\left[c_{\mathrm{PMEP}, 0}, c_{\mathrm{PMEP}, 1}\right]
\end{aligned}
$$

The resulting fit is shown in Table 3.

The friction torque is estimated using data set B (Table 1). The parameterization was done by minimizing the following error function:

$$
\begin{aligned}
e_{k, \mathrm{FMEP}} & =\left(\mathrm{FMEP}_{\mathrm{data}, k}-\mathrm{FMEP}_{\text {model }, k}\left(\theta_{\mathrm{FMEP}}\right)\right)^{2} \\
\theta_{\mathrm{FMEP}} & =\left[c_{\mathrm{f}, 0}, c_{\mathrm{f}, 1}, c_{\mathrm{f}, 2}, c_{\mathrm{f}, 3}\right]
\end{aligned}
$$

The resulting fit is shown in Table 3.

For the model describing the exhaust manifold temperature, the loss function is calculated as:

$$
\begin{aligned}
& e_{k, T}=\left(T_{\mathrm{em}, \text { data }, k}-T_{\mathrm{em}, \text { model }, k}\left(\theta_{T_{\mathrm{em}}}\right)\right)^{2} \\
& \theta_{T_{\mathrm{em}}}=\left[\eta_{\mathrm{sc}}, c_{\mathrm{em}, \mathrm{h}}\right]
\end{aligned}
$$

The resulting fit is shown in Table 2 .

\begin{tabular}{ccccc}
\hline & $\bar{e}$ & $e_{\sigma}$ & $\bar{e}_{\text {rel }}$ & $e_{\text {rel }, \sigma}$ \\
\hline$\eta_{\text {vol }}$ & $0.84 \%$ & $0.61 \%$ & $0.92 \%$ & $0.69 \%$ \\
$T_{\mathrm{em}}$ & $5.20 \mathrm{~K}$ & $3.40 \mathrm{~K}$ & $0.78 \%$ & $0.49 \%$ \\
\hline
\end{tabular}

Table 2: Cylinder model fit to dataset A. $\bar{e}$ is the mean absolute error, $e_{\sigma}$ the variance of the absolute error, $\bar{e}_{\text {rel }}$ the relative error, and $e_{\text {rel }, \sigma}$ the variance of the relative error.

\begin{tabular}{lcccc}
\hline & $\bar{e}$ & $e_{\sigma}$ & $\bar{e}_{\text {rel }}$ & $e_{\text {rel }, \sigma}$ \\
\hline IMEP & $24.0 \mathrm{kPa}$ & $11.7 \mathrm{kPa}$ & $1.57 \%$ & $0.42 \%$ \\
PMEP & $99.2 \mathrm{kPa}$ & $105.1 \mathrm{kPa}$ & $23.0 \%$ & $13.6 \%$ \\
FMEP & $0.75 \mathrm{kPa}$ & $0.52 \mathrm{kPa}$ & $0.70 \%$ & $0.43 \%$ \\
\hline
\end{tabular}

Table 3: Cylinder model fit to dataset B. $\bar{e}$ is the mean absolute error, $e_{\sigma}$ the variance of the absolute error, $\bar{e}_{\text {rel }}$ the relative error, and $e_{\text {rel }, \sigma}$ the variance of the relative error.

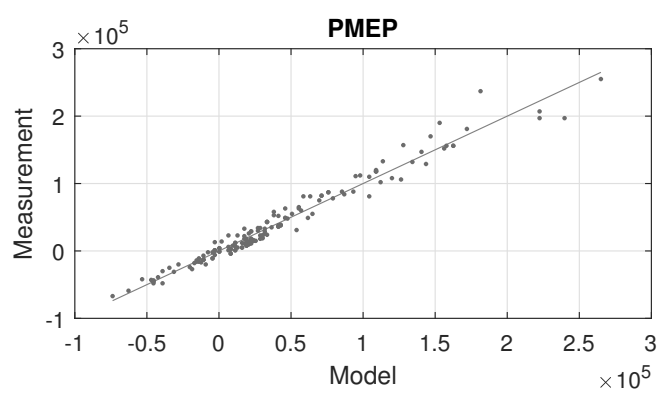

Figure 3: PMEP fit to dataset $B$. Red line shows the one-to-one ratio.

\subsection{Turbocharger}

The turbocharger dynamics is modeled from Newton's second law of motion according to:

$$
\frac{d}{d t} \omega_{\mathrm{tc}}(t)=\frac{P_{\mathrm{t}} \eta_{\mathrm{m}}(t)-P_{\mathrm{c}}(t)}{\omega_{\mathrm{tc}}(t) J_{\mathrm{tc}}}
$$

Parameters. There is one parameter to estimate: $J_{\mathrm{tc}}$. The validation is seen in Section 5.

\subsection{Turbine}

The turbine power, including the mechanical efficiency of the turbocharger shaft is calculated according to:

$$
\begin{aligned}
P_{\mathrm{t}} \eta_{\mathrm{m}}(t) & =W_{\mathrm{t}}(t) c_{\mathrm{p}, \mathrm{e}} T_{\mathrm{em}}(t) \eta_{\mathrm{t}}(t)\left(1-\Pi_{\mathrm{t}}(t)^{1-1 / \gamma_{\mathrm{e}}}\right) \\
\Pi_{\mathrm{t}}(t) & =\frac{p_{\mathrm{ats}}}{p_{\mathrm{em}}(t)}
\end{aligned}
$$

where $p_{\text {ats }}$ is the pressure in the exhaust aftertreatment system. For the flow, the square root turbine flow model in [3] is adapted to to describe dataset D:

$$
W_{\mathrm{t}, \mathrm{corr}}(t)=k_{0}(t)\left(1-\Pi_{\mathrm{t}}(t)^{k_{1}(t)}\right)^{k_{2}(t)}
$$




$$
\begin{aligned}
k_{0}(t) & =c_{00}+c_{02} N_{\mathrm{tc}, \mathrm{corr}, \mathrm{I}}^{2}(t) \\
k_{1}(t) & =c_{10}+c_{11} N_{\mathrm{tc}, \mathrm{corr}, \mathrm{I}}(t) \\
k_{2}(t) & =c_{20}+c_{21} N_{\mathrm{tc}, \mathrm{corr}, \mathrm{I}}(t)+c_{22} N_{\mathrm{tc}, \mathrm{corr}, \mathrm{I}}^{2}(t) \\
N_{\mathrm{tc}, \mathrm{corr}, \mathrm{I}}(t) & =\frac{\omega_{\mathrm{tc}}(t)}{\sqrt{T_{e m}(t)}} \frac{30}{\pi} \frac{1}{1000}
\end{aligned}
$$

The turbine mass flow is calculated as:

$$
W_{\mathrm{t}}(t)=W_{\mathrm{t}, \mathrm{corr}}(t) \frac{p_{\mathrm{em}} 10^{-3}}{\sqrt{T_{\mathrm{em}}}}
$$

For the turbine efficiency, the standard approach of modeling it from the blade-speed-ratio (BSR) is taken. However, since the BSR lines do not overlap in the $\mathrm{BSR}-\eta$ plane in dataset $\mathrm{D}$, a speed dependence is included. The model is described by:

$$
\begin{aligned}
& \eta_{t}(t)=\eta_{t, \max }(t)-k_{\eta}(t)\left(\mathrm{BSR}(t)-\mathrm{BSR}_{\mathrm{opt}}(t)\right)^{2} \\
& \operatorname{BSR}(t)= \frac{\omega_{\mathrm{tc}}(t) D_{\mathrm{t}} / 2}{\sqrt{2 c_{\mathrm{p}, \mathrm{e}} T_{\mathrm{em}}(t)\left(1-\Pi_{\mathrm{t}}(t){ }^{1-\frac{1}{\gamma_{\mathrm{e}}}}\right)}} \\
& \operatorname{BSR}_{\mathrm{opt}}(t)= c_{\mathrm{BSR}, 0}+c_{\mathrm{BSR}, 1} N_{\mathrm{tc}, \mathrm{corr}, \mathrm{II}}(t)+ \\
& \eta_{\mathrm{t}, \mathrm{max}}(t) c_{\mathrm{BSR}, 2} N_{\mathrm{tc}, \mathrm{corr}, \mathrm{II}}(t) \\
& k_{\eta}(t)= c_{\eta_{t}, 0}+c_{\eta_{t}, 1} N_{\mathrm{tc}, \mathrm{corr}, \mathrm{II}}+(t) \\
& N_{\mathrm{tc}, \mathrm{corr}, \mathrm{II}}(t)=\frac{\omega_{\mathrm{tc}}(t)}{\sqrt{T_{e m}(t)} \frac{30}{\pi} \frac{1}{10000} N_{\mathrm{tc}, \mathrm{corr}, \mathrm{II}}(t)}
\end{aligned}
$$

Parameters. There are 14 parameters to estimate: $c_{00}, c_{02}, c_{10}, c_{11}, c_{20}, c_{21}, c_{22}, c_{\mathrm{BSR}, 0}, c_{\mathrm{BSR}, 1}, c_{\mathrm{BSR}, 2}$, $c_{\eta_{t}, 0}, c_{\eta_{t}, 1}, c_{\max , 0}$, and $c_{\max , 1}$.

Parametrization and Validation. The parameterization is carried out by parameterizing the mass flow model and efficiency model separately using dataset D. For the mass flow, the loss function is calculated as:

$$
\begin{aligned}
& e_{k, W \mathrm{t}}=\left(W_{\mathrm{t}, \text { data, } k}-W_{\mathrm{t}, \text { model }, k}\left(\theta_{W \mathrm{t}}\right)\right)^{2}
\end{aligned}
$$

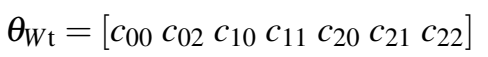

The model fit is shown in Table 4. For the efficiency, the following loss function is used:

$$
e_{k, \eta \mathrm{t}}=\left(\eta_{\mathrm{t}, \text { data }, k}-\eta_{\mathrm{t}, \text { model }, k}\left(\theta_{\eta \mathrm{t}}\right)\right)^{2}
$$

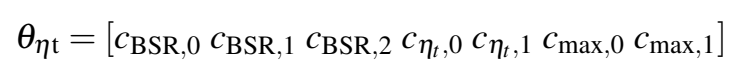

The model fit is shown in Table 4.

\subsection{Wastegate}

The wastegate is developed in the same way as the throttle (see section 3.2), apart from $\gamma_{\mathrm{a}}$ which is replaced by $\gamma_{\mathrm{e}}$. The wastegate mass flow is described by:

$$
W_{\mathrm{wg}}(t)=\frac{p_{\mathrm{em}}(t)}{\sqrt{R_{\mathrm{e}} T_{\mathrm{em}}}} C_{\mathrm{D}, \mathrm{wg}} A_{\mathrm{wg}, \max } u_{\mathrm{wg}}(t) \Psi_{\mathrm{wg}}(t)
$$

where $\Psi_{\mathrm{wg}}(t)$ is similar to (11a), but $\Pi_{\mathrm{thr}}(t)$ is replaced by $\Pi_{\mathrm{wg}}(t) . \Pi_{\mathrm{wg}}(t)$ is defined as in Equation (12), where $\Pi(t)$ is replaced by $\Pi_{t}(t)$ in $(29 b)$.

Parameters There is only one parameter to estimate, $C_{\mathrm{D}, \mathrm{wg}}$. The wastegate area $A_{\mathrm{wg}, \max }$ is determined from measuring the diameter. $C_{\mathrm{D}, \mathrm{wg}}$ is seen as a tuning constant. Since data was not available to parameterize the wastegate as a separate component, the same approach as for the throttle is taken. The modeling of the wastegate and throttle is similar due to both being controllable valves for restricting the gas flow.

\subsection{Compressor}

The parameterization of the compressor was done using dataset $\mathrm{C}$ and by using LiU CPgui [8], which parameterizes a high-order control-oriented compressor model based on the total least squares algorithm. The fit to dataset A is shown in Table 5.

\section{Full System Parametrization}

An approach taken in [11] and [10] is to refit the parameters to the measurements when all model components

\begin{tabular}{ccccc}
\hline & $\bar{e}$ & $e_{\sigma}$ & $\bar{e}_{\text {rel }}$ & $e_{\text {rel }, \sigma}$ \\
\hline$W_{\mathrm{t}, \mathrm{corr}}$ & $5.1410^{-4}$ & $3.5610^{-4}$ & $1.30 \%$ & $0.95 \%$ \\
& $\mathrm{~kg} / \mathrm{s} \sqrt{\mathrm{K}} / \mathrm{kPa}$ & $\mathrm{kg} / \mathrm{s} \sqrt{\mathrm{K}} / \mathrm{kPa}$ & & \\
$\eta_{\mathrm{t}}$ & $0.92 \%$ & $0.99 \%$ & $1.34 \%$ & $1.47 \%$ \\
\hline
\end{tabular}

Table 4: Turbine model fit to dataset D. $\bar{e}$ is the mean absolute error, $e_{\sigma}$ the variance of the absolute error, $\bar{e}_{\text {rel }}$ the relative error, and $e_{\text {rel }, \sigma}$ the variance of the relative error. 


\begin{tabular}{ccccc}
\hline & $\bar{e}$ & $e_{\sigma}$ & $\bar{e}_{\text {rel }}$ & $e_{\text {rel }, \sigma}$ \\
\hline$W_{\mathrm{c}}$ & $1.0310^{-4}$ & $8.2910^{-4}$ & $0.13 \%$ & $1.51 \%$ \\
& $\mathrm{~kg} / \mathrm{s}$ & $\mathrm{kg} / \mathrm{s}$ & & \\
$\eta_{\mathrm{c}}$ & $0.92 \%$ & $0.99 \%$ & $1.34 \%$ & $1.47 \%$ \\
\hline
\end{tabular}

Table 5: Compressor model fit to dataset A. $\bar{e}$ is the mean absolute error, $e_{\sigma}$ the variance of the absolute error, $\bar{e}_{\text {rel }}$ the relative error, and $e_{\text {rel }, \sigma}$ the variance of the relative error.

are assembled in order to achieve good fit. A similar approach is taken here, but with a slightly different cost function (see equation 2) where a regularization technique is used to limit the changes in parameter values, in order to preserve the model structure. This procedure is divided into two steps. The first step is to refit the torque model to dataset $\mathrm{A}$. The second step is to tune all parameters influencing the steady state levels of the states when the complete model is fully assembled.

\subsection{Torque model}

For the torque model, the following parameters are refitted:

$$
\begin{array}{r}
\theta_{\mathrm{M}}=\left[c_{\mathrm{cal}, 0}, c_{\mathrm{cal}, 10}, c_{\mathrm{cal}, 11}, c_{\mathrm{cal}, 20}, c_{\mathrm{cal}, 21}, c_{\mathrm{cal}, 22},\right. \\
\left.c_{\mathrm{f}, 0}, c_{\mathrm{f}, 1}, c_{\mathrm{f}, 2}, c_{\mathrm{f}, 3}, c_{\mathrm{PMEP}, 0}, c_{\mathrm{PMEP}, 1}\right]
\end{array}
$$

using criterion (2). The resulting fit is shown in Table 6 .

\begin{tabular}{ccccc}
\hline & $\bar{e}$ & $e_{\sigma}$ & $\bar{e}_{\text {rel }}$ & $e_{\text {rel }, \sigma}$ \\
\hline$M_{\text {ice }}$ & $8.42 \mathrm{Nm}$ & $6.59 \mathrm{Nm}$ & $1.81 \%$ & $3.05 \%$ \\
\hline
\end{tabular}

Table 6: Torque model refit to dataset A. $\bar{e}$ is the mean absolute error, $e_{\sigma}$ the variance of the absolute error, $\bar{e}_{\text {rel }}$ the relative error, and $e_{\text {rel }, \sigma}$ the variance of the relative error.

\subsection{Tuning of steady-state levels}

In the same manner as for the torque model, using criterion (2), the parameters influencing the steady-state levels of the states are re-parameterized. To do this, the full model is simulated and the wastegate is controlled, using a $P I D$-controller to minimize the error:

$$
e_{\mathrm{wg}}=W_{\mathrm{c}}-W_{\mathrm{c}, \mathrm{ref}}
$$

where the compressor massflow reference $W_{\mathrm{c}, \text { ref }}$ is taken from the massflow measurment in dataset $\mathrm{A}$. The result- ing fit is shown in Table 7.

\begin{tabular}{ccccc}
\hline & $\bar{e}$ & $e_{\sigma}$ & $\bar{e}_{\text {rel }}$ & $e_{\text {rel }, \sigma}$ \\
\hline$p_{\mathrm{c}}$ & $2.78 \mathrm{kPa}$ & $4.00 \mathrm{kPa}$ & $1.89 \%$ & $2.80 \%$ \\
$p_{\mathrm{im}}$ & $2.76 \mathrm{kPa}$ & $3.96 \mathrm{kPa}$ & $1.89 \%$ & $2.79 \%$ \\
$p_{\mathrm{em}}$ & $14.73 \mathrm{kPa}$ & $3.96 \mathrm{kPa}$ & $7.33 \%$ & $3.69 \%$ \\
$\omega_{\mathrm{tc}}$ & $1.98 \mathrm{kRPM}$ & $2.42 \mathrm{kRPM}$ & $4.88 \%$ & $7.93 \%$ \\
\hline
\end{tabular}

Table 7: Steady-state levels of states fit to dataset A for the fully parameterized model. $\bar{e}$ is the mean absolute error, $e_{\sigma}$ the variance of the absolute error, $\bar{e}_{\text {rel }}$ the relative error, and $e_{\mathrm{rel}, \sigma}$ the variance of the relative error.

\section{Full System Validation}

The complete system model is simulated with the control signals recorded from a dynamic engine test (part of data set E). The result from the simulated system, in comparison with the measurement data is displayed in Figure 4.

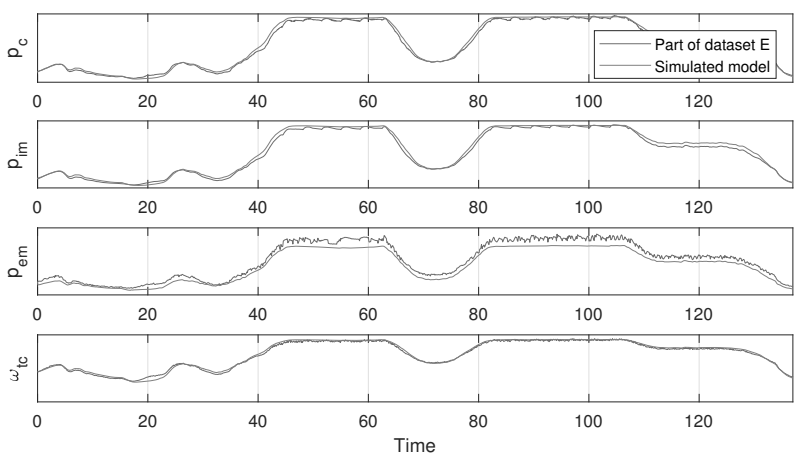

Figure 4: State validation, simulated engine model compared to dynamic measurement data. The control signals are taken from measurements.

\section{Conclusions}

A mean value engine model of a Scania 13 liters heavyduty diesel engine has been developed and validated using both stationary and dynamic measurements. The results show good agreement with measurements, showing that both dynamics and steady-state levels are well represented indicating that the model is well suited for studying the engine dynamics and fuel optimal control. The model is open-source and downloadable from www.fs.isy.liu.se/Software/. 


\section{References}

[1] Ekberg K, Leek V, Eriksson L. Optimal control of wastegate throttle and fuel injection for a heavy-duty turbocharged diesel engine during tip-in. In 58th Conference on Simulation and Modelling (SIMS 58). Reykjavik, Iceland, 2017.

[2] Eriksson L. Mean value models for exhaust system temperatures. SAE 2002 World Congress \& Exhibition, 2002.

[3] Eriksson L, Nielsen L. Modeling and Control of Engines and Drivelines. John Wiley and Sons Ltd, 2014.

[4] Gamma Technologies. GT-Power User's Manual. GTSuite Version 6.1, 2004.

[5] Heywood JB. Internal Combustion Engine Fundamentals. McGraw-Hill, 1988.

[6] Holmbom R, Eriksson L. Analysis and development of compact models for mass flows through butterfly throttle valves. In WCX World Congress Experience. SAE International, Apr 2018.
[7] Llamas X, Eriksson L. Control-oriented compressor model with adiabatic efficiency extrapolation. $S A E$ International Journal of Engines, 10(4), 2017.

[8] Llamas X, Eriksson L. LiU CPgui: A toolbox for parameterizing compressor models. Technical Report LiTH-ISY-R-3102, Department of Electrical Engineering, Linköpings Universitet, SE-581 83 Linköping, Sweden, 2018.

[9] Shen T, Ohata A. Modeling and Control Design for Automotive Engines - with MATLAB Engine Simulator CD-ROM. ISBN 978e-4-339-04610-6, 2011.

[10] Sivertsson M, Eriksson L. Modeling for Optimal Control: A Validated Diesel-Electric Powertrain Model. SIMS 2014 - 55th International Conference on Simulation and Modelling, Ålborg, Denmark, 2011.

[11] Wahlström J, Eriksson L. Modelling diesel engines with a variable-geometry turbocharger and exhaust gas recirculation by optimization of model parameters for capturing non-linear system dynamics. Proceedings of the Institution of Mechanical Engineers, Part D: Journal of Automobile Engineering, 225(7):960-986, 2011. 